\title{
Blinded Treatment Epoch
}

National Cancer Institute

\section{Source}

National Cancer Institute. Blinded Treatment Epoch. NCI Thesaurus. Code C102255.

A period in a clinical study during which subjects receive blinded therapeutic treatment. 